\title{
Application of sensitivity analysis for the design of six-degree-of-freedom measurement system
}

\author{
Jong-Ahn Kim \\ Eui Won Bae \\ Soo Hyun Kim \\ Yoon Keun Kwak \\ Korea Advanced Institute of Science and \\ Technology \\ Department of Mechanical Engineering \\ 373-1, Kusong-dong, Yusong-gu \\ Taejon 305-701 \\ Republic of Korea \\ E-mail: kyk@kaist.ac.kr
}

\begin{abstract}
We present a sensitivity analysis and performance evaluation of a six-degree-of-freedom measurement system that uses a diffraction grating as a cooperative target. To design the measurement system, we require a theoretical analysis of performance, such as sensitivity of each sensing direction. The intensity distributions of the diffracted beams are calculated with the scalar diffraction theory and sensitivity of the measurement system is analyzed against the variations of design parameter values. Using the results of sensitivity analysis, we design the measurement system and evaluate its performance with resolution, measurement error, and crosstalk. The resolution is less than $0.2 \mu \mathrm{m}$ in translation and 0.5 arcsec in rotation. In experiments, measurement error and crosstalk between sensing channels are within $\pm 0.5 \mu \mathrm{m}$ in translation and \pm 2 arcsec in rotation. (c) 2001 Society of Photo-Optical Instrumentation Engineers. [DOI: $10.1117 / 1.1418714]$
\end{abstract}

Subject terms: sensitivity analysis; scalar diffraction theory; Fourier transforms; diffraction gratings; quadrant detectors.

Paper 010021 received Jan. 19, 2001; revised manuscript received May 23, 2001 and July 5, 2001; accepted for publication July 6, 2001.

\section{Introduction}

A six-degree-of-freedom (DOF) measurement system is required in various areas such as control of precision machines, precision assembly, and vibration analysis to obtain the information of three-translational and three-rotational motions. There are two ways to measure six-DOF motion of a rigid body. One is using multiple interferometers for each axis ${ }^{1}$ and the other is detecting the movement of beams reflected from cooperative targets. ${ }^{2-4}$ Although the first method can obtain high resolution and decoupled information for each axis, it increases the size and complexity of a system and requires a high-quality reference mirror. Hence it is usually applied to control and calibration of precision stages. For the second method, it is difficult to fabricate a miniaturized reflective target ${ }^{2}$ precisely and to measure the motion of a small object because it needs sufficient space for multiple reflective $\operatorname{targets}^{3}$ or optical devices. ${ }^{4}$

A new six-DOF measurement system using a diffraction grating target was proposed to overcome these problems. ${ }^{5}$ Since this system uses several diffracted beams generated from a diffraction grating, it requires only one laser source and one cooperative target, which can simplify the structure of the measurement system. In addition, a diffraction grating can be produced through a microfabrication process or replication, and therefore this system can measure the displacement of a miniaturized object without any complicated devices.

To design a six-DOF measurement system, theoretical analysis of performance is required. Among several perfor- mance indices, sensitivity is an important factor that determines the resolution of a measurement system. In addition to increasing the sensitivity of each sensing direction, it is also important to equalize the sensitivity of each measurement direction in designing a multi-DOF measurement system. Therefore it is essential to develop a method that relates the variations of design parameter values and sensitivity of each sensing direction. Based on the results of those analyses, the values of design parameters can be found that optimize the performance of a measurement system.

There were several previous works to enhance the performance of measurement systems through sensitivity analysis. Some works proposed analytic methods to improve the performance of detecting systems for atomic force microscopy (AFM). This system, based on the intensity distribution of beams, used the values of design parameters calculated from the optimization of detection sensitivity. ${ }^{6,7}$ Other research presented a new method to increase the resolution of optical beam deflection method (OBDM) with a passive interferometer and Ronchi grating. ${ }^{8}$ However, these are only single-DOF measurement systems and new analytic methods are required for a sixDOF measurement system.

This paper analyzes the intensity distributions of diffracted beams with the scalar diffraction theory and compares the theoretical results with the experimental ones. Sensitivity analysis using these intensity distributions is discussed. We construct a six-DOF measurement system using design parameter values determined from the sensitivity analysis and evaluate its performance. 
Kim et al.: Application of sensitivity analysis ...

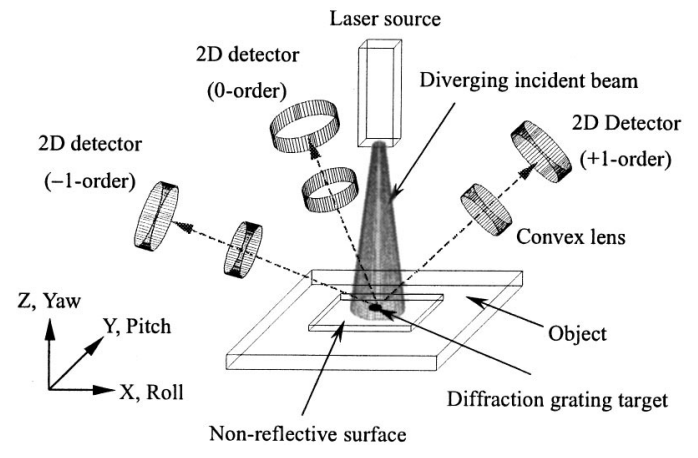

Fig. 1 Configuration of the six-DOF measurement system.

\section{Six-DOF Measurement System}

The six-DOF measurement system consists of a laser source, a diffraction grating target, three convex lenses, and three 2-D detectors, that sense the movement of three diffracted beams, $+1,0,-1$ order diffracted beams (Fig. 1). A monochromatic incident beam is diffracted into discrete directions from a diffraction grating and their directions are calculated using the grating equation. When a miniaturized diffraction grating, which acts as a small reflective target, moves inside a spherically diverging incident beam that has a larger diameter than this grating, this movement changes the generating position and propagating directions of diffracted beams. The translation varies the position where the incident beam is reflected [Fig. 2(a)], and the rotation changes the directions of diffracted beams [Fig. 2(b)]. This results in the variation of detecting positions of diffracted beams and the information on six-DOF displacement can be obtained from the coordinates of diffracted beams.

The six-DOF displacement of an object is calculated kinematically from the coordinates of the diffracted beams on the detectors. The calculation process consists of two steps: a forward and an inverse problem-solving step. In the forward step, the coordinates of the diffracted beams on the detectors are estimated. In the inverse step, an actual sixDOF displacement is determined from the coordinates of the diffracted beams. The solutions of the forward problem, which are the coordinates on the detectors, are calculated with the grating equation and ray optics assumption. Since they are obtained as six nonlinear equations, the inverse problem is solved through a numerical iterative method, Newton's method. The detailed mathematical equations were explained in the reference paper. ${ }^{5}$

In this measurement system, a quadrant photodiode (QPD) detects the 2-D movement of the diffracted beam. Two axial outputs of the Q-PD are the difference signals calculated from the voltage outputs proportional to the intensity of an incident beam on each photodiode cell, and so affected by the intensity distribution and power of the incident beam. Therefore, to analyze the sensitivity of the sixDOF measurement system, we need not only kinematic analysis that derives the relationship between the coordinates of the diffracted beams and input displacement, but also optical analysis that calculates the intensity distributions of the diffracted beams.

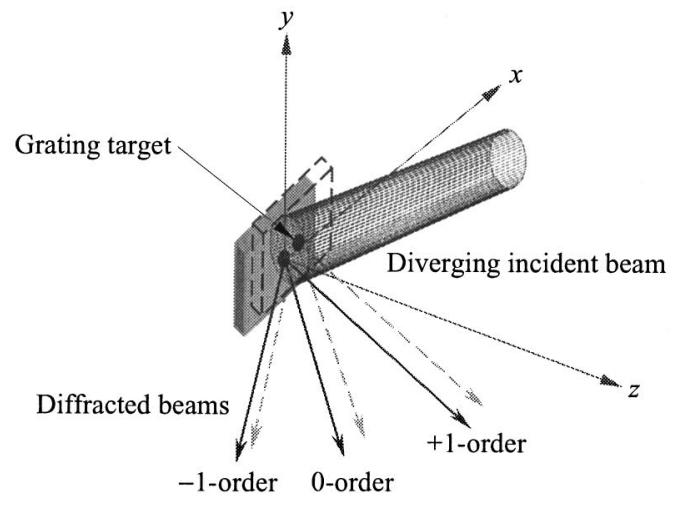

(a)

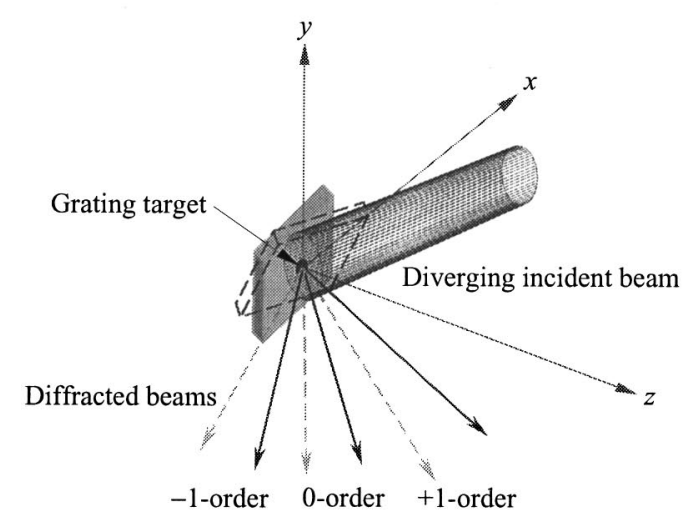

(b)

Fig. 2 Principle of six-DOF measurement: (a) variation of the generating position of diffracted beams (3-D translation) and (b) variation of the propagating directions of diffracted beams (3-D rotation).

\section{Intensity Distribution}

Vector diffraction theory is required for the efficiency analysis of a diffraction grating that has a pitch of the order of the wavelength of an incident beam. However, in the proposed system, a more simplified method, scalar diffraction theory, can be applied since the polarization direction of the incident beam is fixed and the analysis of diffraction patterns generated from a cooperative target is the main focus. $^{9}$

Coordinate systems for analysis of the $m$-order diffracted beam are defined as the grating coordinate system $\left\{G_{m}\right\}$, the lens coordinate system $\left\{L_{m}\right\}$, and the detector coordinate system $\left\{D_{m}\right\}$, where $m$ is the order of the diffracted beam. The origin of $\left\{G_{m}\right\}$ is located at the center of the diffraction grating target, the $y$ axis is parallel with the grating pitch and the $z$ axis is set as the optical axis, the propagating direction of the diffracted beam. The origin of $\left\{L_{m}\right\}$ is apart from that of $\left\{G_{m}\right\}$ by $Z_{m l}$ on the $z$ axis, which also coincides with the optical axis. Note $\left\{D_{m}\right\}$ is parallel to $\left\{L_{m}\right\}$ and separated from $\left\{L_{m}\right\}$ by $Z_{m d}$ on the $z$ axis. Here, $\theta_{m}$ denotes the angle between the incident beam and each diffracted beam (Fig. 3).

Based on the definition of coordinate systems, the intensity distribution of the zero-order diffracted beam is analyzed. When there is a Gaussian beam incident on the diffraction grating and the waist of the beam is apart from 


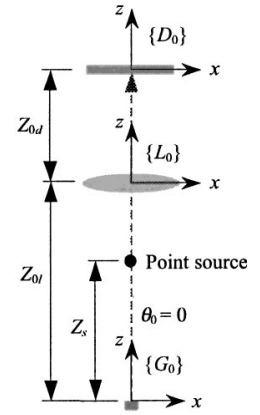

(a)

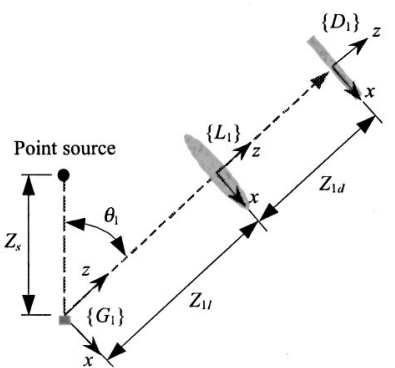

(b)
Fig. 3 Analysis model for the computation of intensity distributions: (a) 0 -order and (b) \pm 1 -order diffracted beam.

$\left\{G_{0}\right\}$ by $Z_{s}$, it is possible to assume a spherical wave is generated from a point source if the diffraction grating is separated sufficiently from the beam waist. ${ }^{10}$ From this assumption, the electric field on the diffraction grating $U_{i}$ can be expressed as

$U_{i}\left(x_{g}, y_{g}\right)=U_{0} \exp \left(-\frac{x_{g}^{2}+y_{g}^{2}}{w_{b}^{2}}\right) \frac{\exp \left(j k r_{s g}\right)}{r_{s g}}$,

where $x_{g}$ and $y_{g}$ are coordinates on the diffraction grating; $r_{s g}$ is the magnitude of $\mathbf{r}_{s g}$, which is a vector from the point source to the diffraction grating; $U_{0}$ is a constant proportional to the power of the incident beam; and $w_{b}$ is the radius of the incident beam. The sinusoidal diffraction grating modifies the electric field $U_{i}$ as

$U_{0 g}\left(x_{g}, y_{g}\right)=U_{i}\left(x_{g}, y_{g}\right) \exp \left(j k \Delta_{0}\right)$,

$\Delta_{0}=a\left(\cos \theta_{s g}+\cos \theta_{0 l}\right) \sin \left(2 \pi f_{g} x_{g}\right)$,

where $k \Delta_{0}$ is a phase change of electric field; $a$ and $f_{g}$ are groove amplitude and pitch of the diffraction grating; $\theta_{s g}$ is the angle between $\mathbf{r}_{s g}$ and $n_{g}$, which is a unit normal vector to the grating surface; $\theta_{0 l}$ denotes the angle between $n_{g}$ and $\mathbf{r}_{0 l}$, which is a vector from the grating to the convex lens. Hence, using Eq. (2) and the Huygens-Fresnel principle, the electric field on the convex lens $U_{0 l}$ is formulated as

$$
\begin{aligned}
U_{0 l}\left(x_{0 l}, y_{0 l}\right)= & \frac{1}{j \lambda} \int_{-\infty}^{\infty} \int_{-\infty}^{\infty} \operatorname{circ}(r) \\
& \cdot U_{0 g}\left(x_{g}, y_{g}\right) \frac{\exp \left(j k r_{0 l}\right)}{r_{0 l}} \cos \theta_{0 l} \mathrm{~d} x_{g} \mathrm{~d} y_{g},
\end{aligned}
$$

$\operatorname{circ}(r)=\left\{\begin{array}{ll}1 & \text { for } r \leqslant 1 \\ 0 & \text { otherwise }\end{array}, \quad r=\frac{x_{g}^{2}+y_{g}^{2}}{R^{2}}\right.$,

where $R$ is the radius of the diffraction grating target; $x_{0 l}$ and $y_{0 l}$ are coordinates on the lens surface, and $r_{0 l}$ is the magnitude of $\mathbf{r}_{0 l}$. From the paraxial theorm, we assumed the lens surface as the $x-y$ plane of the lens coordinate system with negligible thickness and Eq. (3) can be simplified using the Fresnel approximation and Eq. (4). In the approximation, we consider that the amplitude of groove is similar to the pitch of grating and the diffracted beams do not interfere with each other since they are separated sufficiently.

$\exp (j \Delta \sin \Phi)=\sum_{m=-\infty}^{\infty} J_{m}(\Delta) \exp (j m \Phi)$,

where $J_{m}(\Delta)$ is the first kind Bessel function and the electric field $U_{0 l}$ is shown as Eq. (5). We can find that Eq. (5) is the Fourier transform of the product of the electric field $U_{0 g}$ and the quadratic-phase exponential term.

$$
\begin{aligned}
U_{0 l}\left(x_{0 l}, y_{0 l}\right) \approx & A_{0 l} \int_{-\infty}^{\infty} \int_{-\infty}^{\infty} \widetilde{U}_{0 g}\left(x_{y}, y_{g}\right) \exp \left[-j 2 \pi\left(f_{x 0 l} x_{g}\right.\right. \\
& \left.\left.+f_{y 0 l} y_{g}\right)\right] \mathrm{d} x_{g} \mathrm{~d} y_{g},
\end{aligned}
$$

$A_{0 l}=\frac{U_{0} J_{0}(2 k a)}{j \lambda Z_{s} Z_{0 l}} \exp \left[j k\left(Z_{s}+Z_{0 l}+\frac{x_{0 l}^{2}+y_{0 l}^{2}}{2 Z_{0 l}}\right)\right]$,

$$
\begin{aligned}
\widetilde{U}_{0 g}\left(x_{g}, y_{g}\right)= & \operatorname{circ}(r) \exp \left[-\frac{\left(x_{g}^{2}+y_{g}^{2}\right)}{w_{b}^{2}}\right] \\
& \times \exp \left[j k \frac{\left(x_{g}^{2}+y_{g}^{2}\right)\left(Z_{s}+Z_{0 l}\right)}{2 Z_{s} Z_{0 l}}\right],
\end{aligned}
$$

$f_{x 0 l}=\frac{x_{0 l}}{\lambda Z_{0 l}}, \quad f_{y 0 l}=\frac{y_{0 l}}{\lambda Z_{0 l}}$.

Now, it is possible to calculate the electric field on the detector. Using Eq. (5) and the Huygens-Fresnel principle, the electric field on the detector surface $U_{0 d}$ is expressed as

$$
\begin{aligned}
U_{0 d}\left(x_{0 d}, y_{0 d}\right)= & \frac{1}{j \lambda} \int_{-\infty}^{\infty} \int_{-\infty}^{\infty} U_{0 l}\left(x_{0 l}, y_{0 l}\right) \\
& \times \exp \left[-j k \frac{\left(x_{0 l}^{2}+y_{0 l}^{2}\right)}{2 f_{0}}\right] \frac{\exp \left(j k r_{0 d}\right)}{r_{0 d}} \\
& \times \cos \theta_{0 d} \mathrm{~d} x_{0 l} \mathrm{~d} y_{0 l},
\end{aligned}
$$

where $f_{0}$ is the focal length of the convex lens; $r_{0 d}$ is the magnitude of $\mathbf{r}_{0 d}$, which is a vector from the lens to the detector; $\theta_{0 d}$ is the angle between $\mathbf{r}_{0 d}$ and $n_{0 l}$, which is a unit normal vector to the lens surface; and $x_{0 d}$ and $y_{0 d}$ denote the coordinates on the zero-order detector. If the Fresnel approximation is applied, the electric field on the detector $U_{0 d}$ can be simplified as Eq. (7) and the intensity distribution is calculated by squaring the absolute value of $U_{0 d}$. 


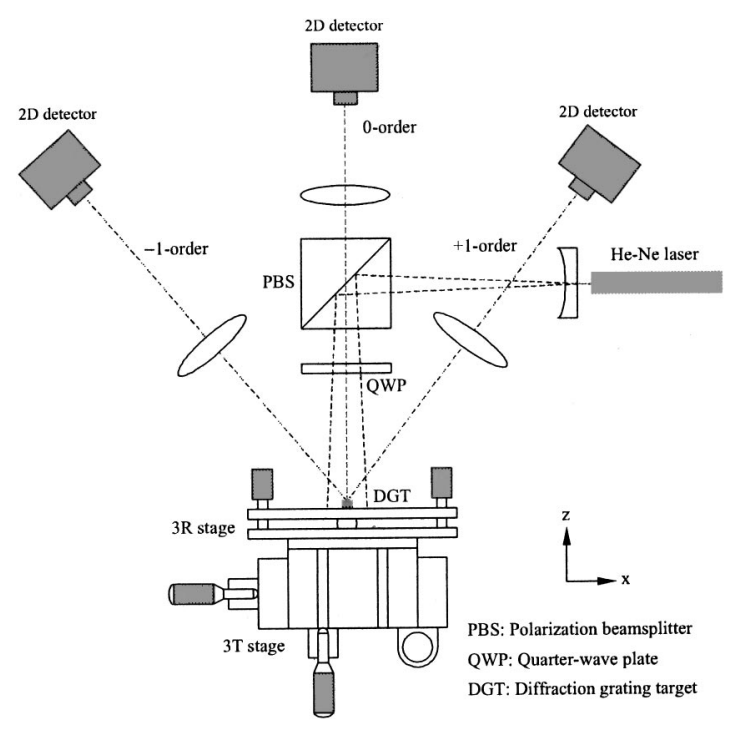

Fig. 4 Experimental setup of the six-DOF measurement system.

$$
\begin{aligned}
& U_{0 d}\left(x_{0 d}, y_{0 d}\right) \approx A_{0 d} \int_{-\infty}^{\infty} \int_{-\infty}^{\infty} \widetilde{U}_{0 l}\left(x_{0 l}, y_{0 l}\right) \\
& \times \exp \left[-j 2 \pi\left(f_{x 0 d} x_{0 l}+f_{y 0 d} y_{0 l}\right)\right] \\
& \times \mathrm{d} x_{0 l} \mathrm{~d} y_{0 l}, \\
& A_{0 d}=-\frac{U_{0} J_{0}(2 k a)}{\lambda^{2} Z_{s} Z_{0 l} Z_{d}}, f_{x 0 d}=\frac{x_{0 d}}{\lambda Z_{0 d}}, f_{y 0 d}=\frac{y_{0 d}}{\lambda Z_{0 d}}, \\
& \tilde{U}_{0 l}\left(x_{0 l}, y_{0 l}\right)= \frac{U_{0 l}\left(x_{0 l}, y_{0 l}\right)}{A_{0 l}} \\
& \times \exp \left[j k \frac{\left(x_{0 l}^{2}+y_{0 l}^{2}\right)}{2}\left(\frac{1}{Z_{0 l}}+\frac{1}{Z_{0 d}}-\frac{1}{f}\right)\right] .
\end{aligned}
$$

The intensity distribution of the \pm 1 -order diffracted beam can be found in a similar way. However, the shape of the grating target should be considered as an ellipse with the $x$ axis as the minor axis, since the propagating direction of the \pm 1 -order diffracted beam is inclined from the unit normal vector of the grating target by $\theta_{i}$ (Fig. 3 ).

To validate the analysis of the intensity distribution drawn from scalar diffraction theory, we compared the analytic results with experimental ones. In the experimental setup, a diffraction grating was attached to a six-DOF stage system and the intensity distribution of each diffracted beam was measured with a 2-D CCD camera. A concave lens and a polarization beamsplitter directed the diverging incident beam normally to the grating target. Each diffracted beam propagated through the convex lens and reached the CCD camera (Fig. 4). Table 1 summarizes the specifications of devices composing the experimental setup. The intensity distribution was measured against the variation of distance between the convex lens and the CCD cam-

\begin{tabular}{|c|c|}
\hline Device & Specification \\
\hline Diffraction grating target & $\begin{array}{l}\text { Pitch: } 1200 \text { grooves } / \mathrm{mm} \\
\text { Diameter: } 0.5 \mathrm{~mm}\end{array}$ \\
\hline Laser source & $\begin{array}{l}\text { Output power: } 2 \mathrm{~mW} \\
\text { Wavelength: } 632.8 \mathrm{~nm}\end{array}$ \\
\hline Concave lens & Focal length: $32.0 \mathrm{~mm}$ \\
\hline Convex lenses & $\begin{aligned} & \text { Focal length: } 25.4 \mathrm{~mm} \text { ( } \pm 1 \text {-order) } \\
& 65.0 \mathrm{~mm} \text { (0-order) }\end{aligned}$ \\
\hline Six-DOF stage system & $\begin{array}{l}\text { Translational resolution: } 0.07 \mu \mathrm{m} \\
\text { Rotational resolution: } 0.14 \text { arcsec }\end{array}$ \\
\hline External displacement sensor & $\begin{array}{l}\text { Resolution: } 0.01 \mu \mathrm{m} \\
\text { Linearity } \pm 0.05 \% \text { of F.S. }\end{array}$ \\
\hline
\end{tabular}
era.

When $Z_{0 l}$, the distance from the diffraction grating to the convex lens, was fixed at $100 \mathrm{~mm}$ and $Z_{0 d}$, the distance from the lens to the CCD camera, was changed, simulation
Table 1 Specifications of devices in the experimental setup.

and experimental results for the zero-order diffracted beam were obtained (Fig. 5). As shown in Fig. 5(a), if the CCD camera was located near to the focal point, the intensity distribution was similar to the Fraunhoffer diffraction pattern of a circular aperture. However, as the distance $Z_{0 d}$ increased, it showed a Fresnel diffraction pattern for the growth of the quadratic-phase exponential term.

Figure 6 shows the intensity distribution of the +1 -order diffracted beam while fixing $Z_{1 l}$ at $85 \mathrm{~mm}$ and changing $Z_{1 d}$. Due to its inclined propagation direction, the shape of the intensity distribution was not circular but elliptical. The
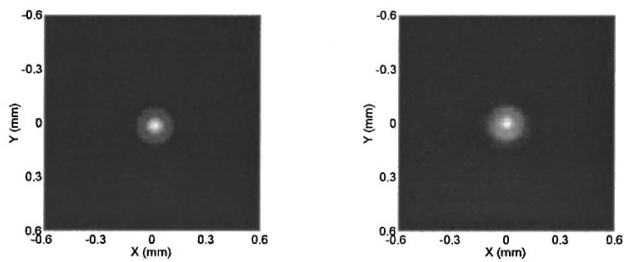

(a)
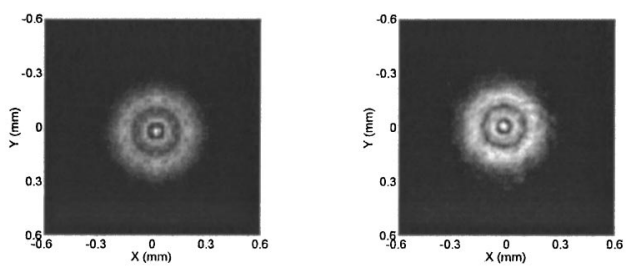

(b)
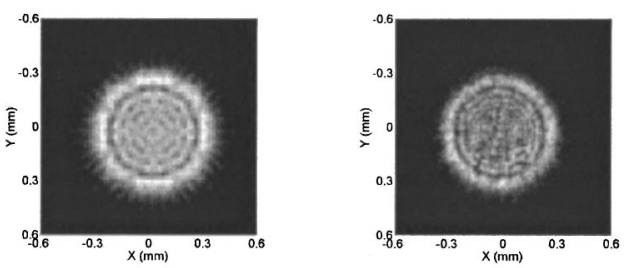

(c)

Fig. 5 Intensity distributions of the zero-order diffracted beam; simulation (left), experiment (right): (a) $Z_{0 d}=100 \mathrm{~mm}$, (b) $Z_{0 d}$ $=140 \mathrm{~mm}$, and (c) $Z_{0 d}=165 \mathrm{~mm}$. 

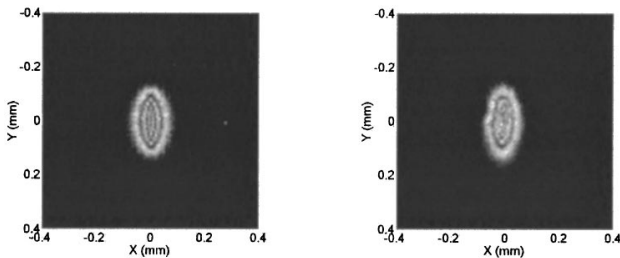

(a)
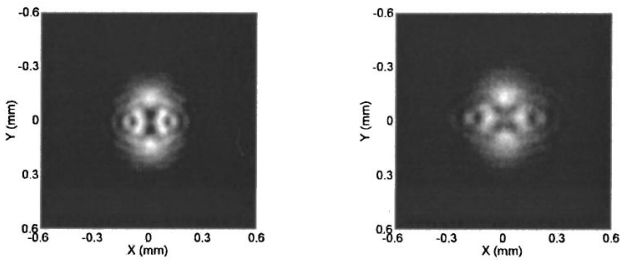

(b)
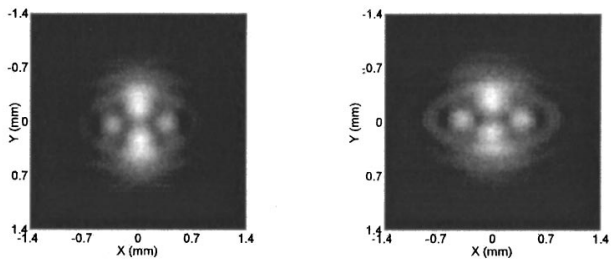

(c)

Fig. 6 Intensity distributions of the +1 -order diffracted beam; simulation (left), experiment (right): (a) $Z_{1 d}=40 \mathrm{~mm}$, (b) $Z_{1 d}=60 \mathrm{~mm}$, and (c) $Z_{1 d}=100 \mathrm{~mm}$.

eccentricity of ellipse increased along the increment of distance $Z_{1 d}$ since diffraction occurs more dominantly in the minor axis of an ellipse.

\section{Sensitivity Analysis}

The variations of detector outputs according to the unit input displacement can be found with the kinematic analysis ${ }^{5}$ and the intensity distributions of the diffracted beams. Each of these values is defined as sensitivity and composes the Jacobian matrix as

$\delta \mathbf{d}=\mathbf{J} \cdot \delta \mathbf{p}, \quad J_{i j}=\frac{\partial d_{i}}{\partial p_{j}}(i=1, \ldots, 6 ; j=1, \ldots, 6)$,

$\delta \mathbf{d}=\left[\delta d_{+1 x} \delta d_{+1 y} \delta d_{0 x} \delta d_{0 y} \delta d_{-1 x} \delta d_{-1 y}\right]^{T}$,

$\delta \mathbf{p}=\left[\begin{array}{llllll}\delta x & \delta y & \delta z & \delta \alpha & \delta \beta & \delta \gamma\end{array}\right]^{T}$,

where $\delta \mathbf{d}$ is an infinitesimal change of the detector output, and $\delta \mathbf{p}$ is an infinitesimal input displacement. The elements of $\delta \mathbf{p}$ are $x, y$, and $z$ axis translation and yaw (rotation about the $z$ axis), pitch (rotation about the $y$ axis), and roll (rotation about the $x$ axis), in order. Since the Jacobian matrix shows overall performance of the measurement system, we can derive principal performance indices from the Jacobian matrix.

To find the relationship between the variations of parameter values and sensitivity, the experimental setup as shown in Fig. 4 was used with three Q-PDs (single element size: $2.5 \times 2.5 \mathrm{~mm}$, gap b/w elements: $30 \mu \mathrm{m})$. The design pa-

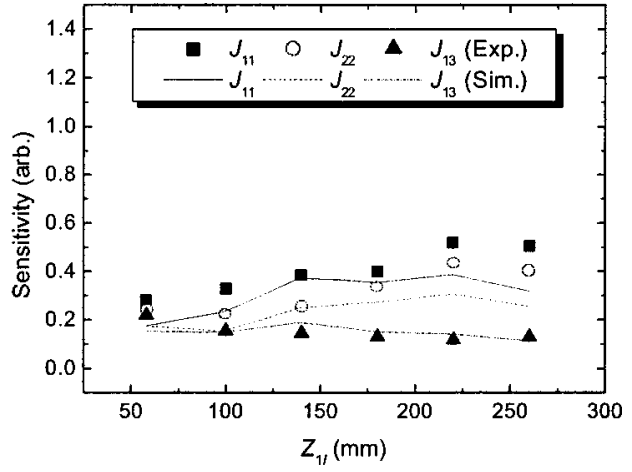

(a)

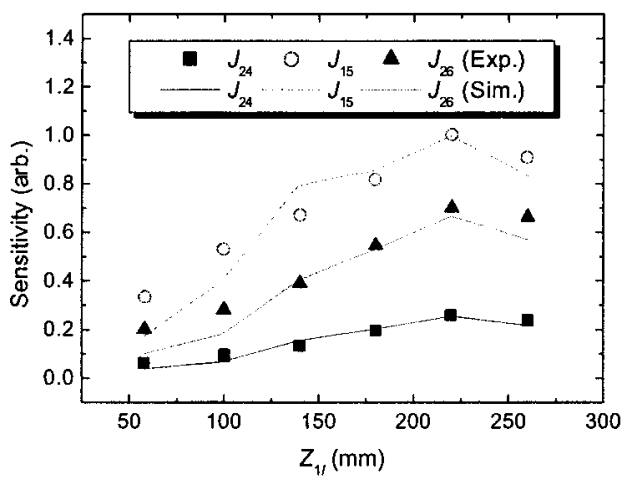

(b)

Fig. 7 Normalized sensitivity for the +1 -order detector against the variation of $Z_{1 /}$ : (a) translational displacement and (b) rotational displacement.

rameters considered in the sensitivity analysis were distances from the grating to the convex lens $\left(Z_{0 l}, Z_{1 l}\right)$ and from the convex lens to the detector $\left(Z_{0 d}, Z_{1 d}\right)$. To analyze the variation of sensitivity, the changes of detector outputs were measured when there were input displacement of 10 $\mu \mathrm{m}$ for translation and $0.01 \mathrm{deg}$ for rotation. In the results, sensitivity was expressed as a component of the Jacobian matrix and normalized by the maximum value of sensitivity obtained for six-DOF displacement input

The sensitivity increased along the increment of $Z_{1 l}$ for reduction of spot size, while fixing the distance from the grating to the +1 -order detector as $300 \mathrm{~mm}$ and changing $Z_{1 l}$. However, when the detector was located near to the image plane, the sensitivity for rotational displacement decreased sharply (Fig. 7).

The variation of sensitivity was less remarkable than in the case of $Z_{1 l}$, when the distance from the grating to the convex lens was $87 \mathrm{~mm}$ and $Z_{1 d}$ changed (Fig. 8). The growth of $Z_{1 d}$ increased spot size as well as movement of the diffracted beam on the detector, therefore overall sensitivity was not affected so much by the variation of $Z_{1 d}$.

In the case of the zero-order detector, we measured the variation of normalized sensitivity for two conditions: distance from the grating to the detector was $370 \mathrm{~mm}$ and $Z_{0 l}$ changed [Fig. 9(a)], distance from the grating to the lens fixed as $85 \mathrm{~mm}$ and $Z_{0 d}$ varied [Fig. 9(b)]. The results for the variation of $Z_{0 l}$ could be explained similarly with the case of $Z_{1 l}$. As $Z_{0 d}$ increased, the detector approached the 


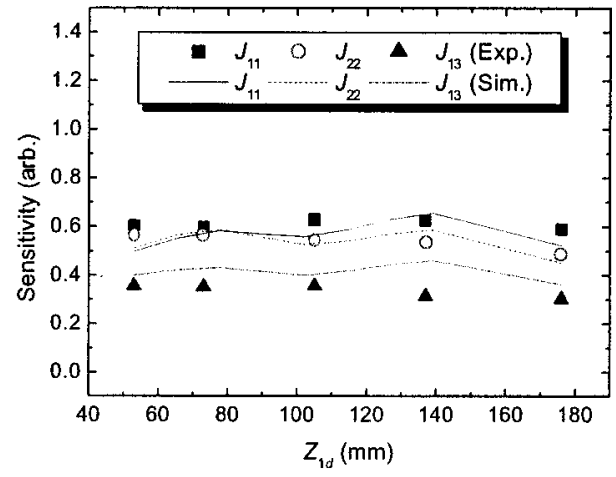

(a)

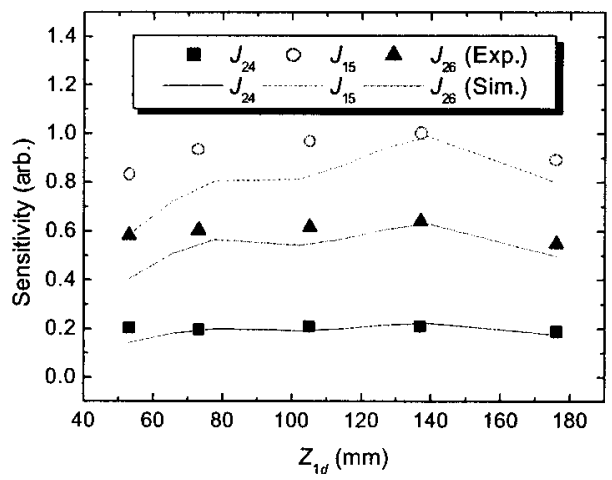

(b)

Fig. 8 Normalized sensitivity for the +1 -order detector against the variation of $Z_{1 d}$ : (a) translational displacement and (b) rotational displacement.

image plane and the sensitivity for rotation decreased. For the same reason as the case of $Z_{1 d}$, the variation of translational sensitivity was less remarkable than that of the rotational.

\section{Performance of the Six-DOF Measurement System}

Considering dimensional constraints and the optimization of performance indices derived from the sensitivity analysis, we can determine the values of design parameters as follows:

$Z_{0 l}=136.4 \mathrm{~mm}, Z_{0 d}=115.2 \mathrm{~mm}, Z_{1 l}=85.0 \mathrm{~mm}$,

$Z_{1 d}=85.0 \mathrm{~mm}$.

The experimental setup was constructed as in Fig. 4 using three Q-PDs. First, power of the diffracted beams and noise level of the detectors were measured. Outputs of the detectors were recorded for $30 \mathrm{~min}$ to calculate the noise level when the diffracted beams were incident on the center of each detector. The power of each diffracted beam was $23.5 \mu \mathrm{W}$ for the 0 -order diffracted beam and $44.5 \mu \mathrm{W}$ for the \pm 1 -order one. The noise level was set as the standard deviation of random noise excluding the drift from mechanical vibration, temperature variation, and laser instabil-

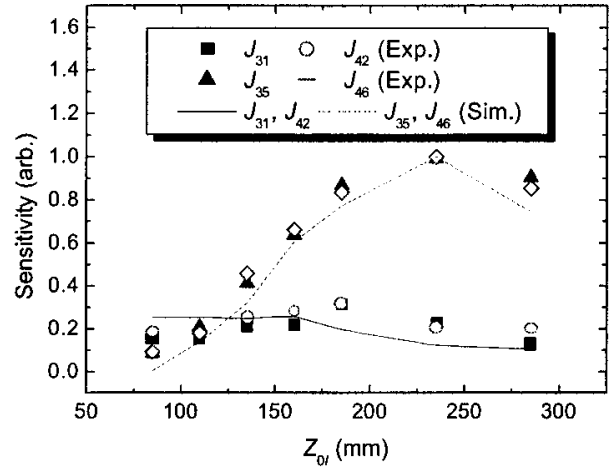

(a)

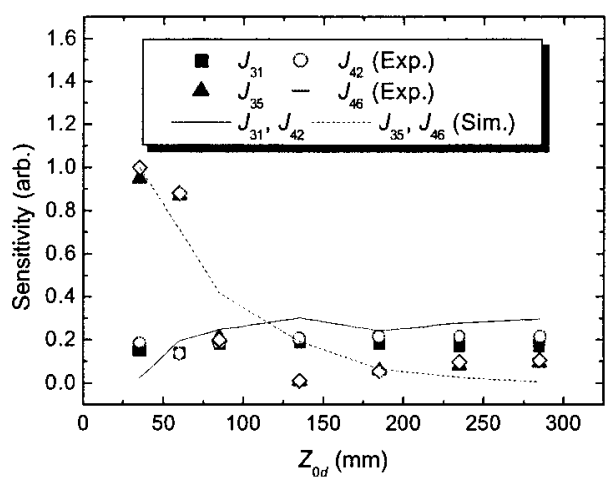

(b)

Fig. 9 Normalized sensitivity for the zero-order detector: (a) variation of $Z_{0 /}$ and (b) variation of $Z_{0 d}$.

ity, because it could be reduced by the improvement of environment. The noise level of each detector was obtained as follows:

$\sigma_{+1 x}=0.0130 \mathrm{~V}, \sigma_{0 x}=0.0052 \mathrm{~V}, \sigma_{-1 x}=0.0127 \mathrm{~V}$,

$\sigma_{+1 y}=0.0073 \mathrm{~V}, \sigma_{0 y}=0.0055 \mathrm{~V}, \sigma_{-1 y}=0.0072 \mathrm{~V}$.

The noise level of the 0 -order detector showed similarities in the $x$ and $y$ axes while each axis of the \pm 1 order detector had different noise levels, since the intensity distribution on the 0 -order detector was circular, but those on the \pm 1 -order detector was elliptical and showed higher intensity on the $y$ axis, as shown in Figs. 5 and 6. This resulted in a higher noise level in the $x$ axis for the \pm 1 -order detector. Thus we can conclude that the intensity distribution affects not only the variation of detector output but also the noise level, which relates to the overall performance of the measurement system.

The changes of output voltage in each detector were measured when input displacement was generated using the six-DOF stage system, which was $10 \mu \mathrm{m}$ for translation and $0.01 \mathrm{deg}$ for rotation. From these values, we calculated sensitivity in each sensing direction and constructed the Jacobian matrix as follows: 


$$
\mathbf{J}=\left[\begin{array}{cccccc}
-0.129 & 0 & 0.057 & 0 & -0.235 & 0 \\
0 & -0.109 & 0 & -0.044 & 0 & 0.149 \\
-0.063 & 0 & 0 & 0 & 0.071 & 0 \\
0 & -0.051 & 0 & 0 & 0 & -0.061 \\
-0.126 & 0 & -0.057 & 0 & -0.231 & 0 \\
0 & -0.108 & 0 & 0.043 & 0 & 0.151
\end{array}\right] \text {, }
$$

where the unit of each element was $\mathrm{V} / \mu \mathrm{m}$ for translation and $\mathrm{V} / 10^{-3} \mathrm{deg}$ for rotation. Using the inverse matrix of $\mathbf{J}$ and the noise level of each detector, the resolution of each sensing direction could be calculated as

$R_{i}=\left[\sum_{j=1}^{6}\left(S_{i j} \sigma_{j}\right)^{2}\right]^{1 / 2}(i=1, \ldots, 6)$,

where $R_{i}$ denotes the resolution of the $i$ 'th sensing direction, $S_{i j}$ is the component of $\mathbf{J}^{-1}$ with $i$ 'th row and $j$ 'th column, and $\sigma_{j}$ is the noise level of $j$ 'th detector output. The resolution of each axis was calculated as follows:

$R_{x}=0.0578 \mu \mathrm{m}, \quad R_{y}=0.0617 \mu \mathrm{m}, \quad R_{z}=0.1584 \mu \mathrm{m}$,

$R_{\alpha}=0.4223 \operatorname{arcsec}, \quad R_{\beta}=0.1325 \operatorname{arcsec}$,

$R_{\gamma}=0.1642$ arcsec

Translation on the $z$ axis and rotation about yaw-axis showed lower resolution than other sensing directions. This can be inferred from the low sensitivity of these measurement axes, as shown in the Jacobian matrix.

To evaluate the accuracy and crosstalk of the measurement system, displacement was generated with a constant step in each measurement direction using the six-DOF stage system. The measurement error, which was the difference between the measured and actual displacement, was recorded at each position. In translation, the output values of the measurement system were taken at every $10 \mu \mathrm{m}$ interval between $\pm 100 \mu \mathrm{m}$. In rotation, 21 output values were measured within the range of \pm 252 arcsec (roll, pitch), and \pm 756 arcsec (yaw), so that the diffracted beams did not get out of the linear ranges of the Q-PDs. The experiment was performed 10 times with the same procedure. The displacement was generated with a differential micrometer and the actual values were obtained from an external displacement sensor. In case of rotation, simple arithmetic using basic trigonometric relations was required additionally to calculate them.

In Fig. 10, data points are the averages of measurement errors obtained at each point in the experiment repeated 10 times and thin lines denote the standard deviation. The errors ranged about $\pm 0.5 \mu \mathrm{m}$ for translation and $\pm 2 \operatorname{arcsec}$ for rotation. The calibration of parameter values and detectors reduced the magnitude of systematic errors remarkably, so they did not increase with displacement, but fluctuated around zero.

The magnitude and trend in the standard deviations of measurement error and crosstalk were similar to the resolution calculated from the noise level (Table 2). Hence we can conclude that the measurement error and crosstalk were caused mostly by random noise of the detector such as shot

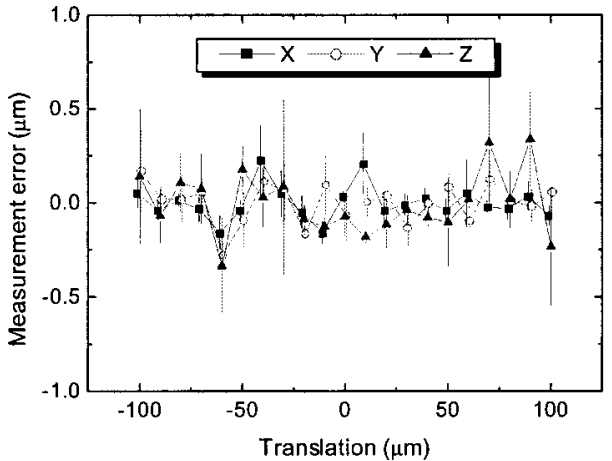

(a)

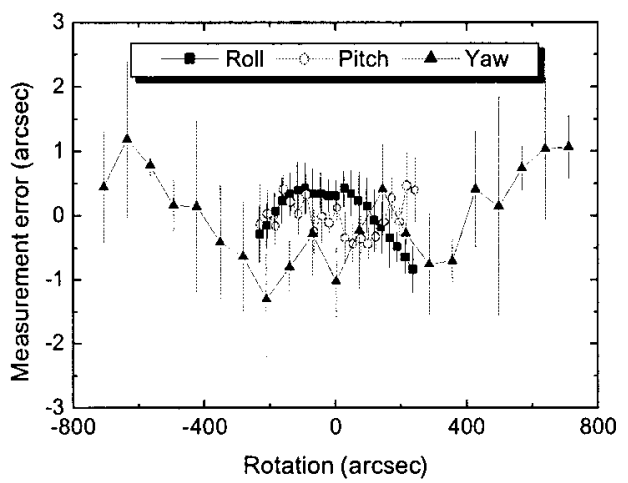

(b)

Fig. 10 Measurement error against the variation of input displacement: (a) translation on the $x, y$, and $z$ axes and (b) rotation about the roll, pitch, and yaw axes.

noise. If a sensing direction, such as $z$ axis translation or yaw-axis rotation, has high uncertainty for its low sensitivity, the measurement error and crosstalk increased since the variation of output was smaller against the same input magnitude and measurement error and crosstalk were larger with the same noise level, conversely.

\section{Conclusions}

This paper presents a design methodology using sensitivity analysis and performance evaluation of the six-DOF measurement system. Scalar diffraction theory was applied to calculate the intensity distribution of the diffracted beam and its validity was proved by comparison with the experimental results. The sensitivity of the measurement system

Table 2 Standard deviations of measurement error and crosstalk for each directional displacement.

\begin{tabular}{ccccccc}
\hline \hline & \multicolumn{6}{c}{ Direction of Input Displacement } \\
\cline { 2 - 7 } & $X$ & $Y$ & $Z$ & Roll & Pitch & Yaw \\
\hline$X(\mu \mathrm{m})$ & $\mathbf{0 . 1 2 2}$ & 0.144 & 0.134 & 0.116 & 0.159 & 0.131 \\
$Y(\mu \mathrm{m})$ & 0.116 & $\mathbf{0 . 1 4 2}$ & 0.123 & 0.172 & 0.120 & 0.135 \\
$Z(\mu \mathrm{m})$ & 0.242 & 0.157 & $\mathbf{0 . 2 4 7}$ & 0.242 & 0.265 & 0.224 \\
Roll (arcsec) & 0.419 & 0.665 & 0.673 & $\mathbf{0 . 4 9 2}$ & 0.441 & 0.501 \\
Pitch (arcsec) & 0.330 & 0.491 & 0.507 & 0.361 & $\mathbf{0 . 4 1 0}$ & 0.474 \\
Yaw (arcsec) & 0.607 & 0.931 & 0.837 & 0.778 & 0.860 & $\mathbf{1 . 0 2 0}$ \\
\hline \hline
\end{tabular}


was analyzed against the variation of design parameter values and the six-DOF measurement system was designed from the results of sensitivity analysis. The resolution of each sensing direction showed $0.2 \mu \mathrm{m}$ in translation and 0.5 arcsec in rotation. In the experiments, the measurement error and crosstalk were within $\pm 0.5 \mu \mathrm{m}$ in translation and \pm 2 arcsec in rotation.

The proposed method for sensitivity analysis can be applied to the design of measurement systems that adopt the OBDM or a reflective target. In this paper, optimal design of the six-DOF measurement system was outlined briefly and details of this subject will be discussed in our subsequent paper. Since performance of the measurement system was directly related to that of the detectors, the accuracy of detectors should be improved to enhance the performance of the measurement system.

\section{Acknowledgments}

This work was supported in part by the Brain Korea 21 Project.

\section{References}

1. K. Fan and M. Chen, "A 6-degree-of-freedom measurement system for the accuracy of X-Y stages," Precis. Eng. 24, 15-23 (2000).

2. E. H. Bokelberg, H. J. Sommer III, and M. W. Trethewey, "A sixdegree-of-freedom laser vibrometer," J. Sound Vib. 178, 643-667 (1994).

3. I. J. Busch-Vishniac, A. B. Buckman, W. Wang, D. Qian, and V Mancevski, "Noncontact position measurement systems using optical sensors," U.S. Patent No. 5367373 (1994)

4. N. K. S. Lee, Y. Cai, and A. Joneja, "High-resolution multidimensional displacement monitoring system," Opt. Eng. 36, 2287-2293 (1997).

5. J. Kim, K. Kim, E. W. Bae, S. Kim, and Y. K. Kwak, "Six-degreeof-freedom displacement measurement system using a diffraction grating," Rev. Sci. Instrum. 71, 3214-3219 (2000).

6. M. G. L. Gustafsson and J. Clarke, "Scanning force microscope springs optimized for optical-beam deflection and with tips made by controlled fracture," J. Appl. Phys. 76, 172-181 (1994).

7. T. E. Schäffer and P. K. Hansma, "Characterization and optimization of the detection sensitivity of an atomic force microscope for small cantilevers," J. Appl. Phys. 84, 4661-4666 (1998).

8. A. García-Valenzuela and R. Díaz-Uribe, "Approach to improve the angle sensitivity and resolution of the optical beam deflection methor using a passive interferometer and a Ronchi grating," Opt. Eng. 36, 1770-1778 (1997).

9. J. W. Goodman, Introduction to Fourier Optics, McGraw-Hill, New York (1996).

10. A. Yariv, Introduction to Optical Electronics, Holt, Rinehart, and Winston, New York (1985)

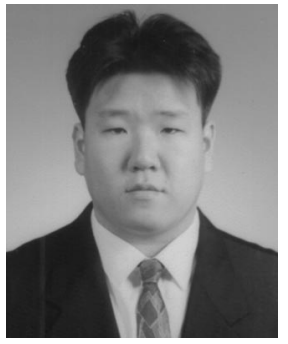

Jong-Ahn Kim is a PhD candidate in the Mechanical Engineering Department, Korea Advanced Institute of Science and Technology (KAIST), Taejon. He received his BS from Hanyang University, Seoul, Korea, in 1995, and his MS from KAIST in 1997. His current interests include optical measurement and micromechatronic system.

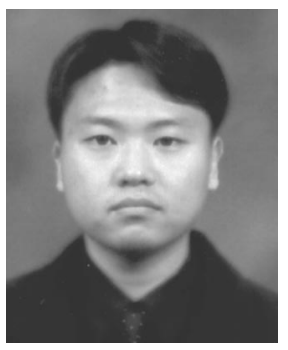

Eui Won Bae received his BS from Korea University, Seoul, in 1999 and his MS from the Mechanical Engineering Department, Korea Advanced Institute of Science and Technology, Taejon, in 2001. His current interests include optomechatronics and evaluation of millistructures.

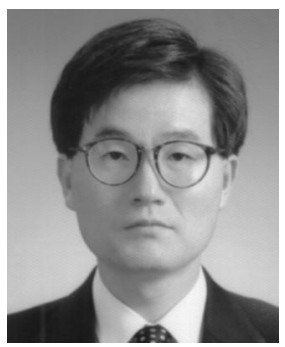

Soo Hyun Kim is a professor with the Mechanical Engineering Department, Korea Advanced Institute of Science and Technology (KAIST), Taejon. He received his MS from KAIST in 1980 and his PhD from the Imperial College, University of London, United Kingdom, in 1991. His research interests include optical measurement, millimachine systems and milliactuation mechanisms.

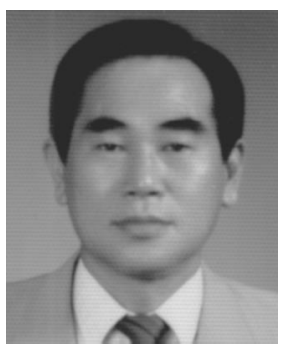

Yoon Keun Kwak is a professor with the Mechanical Engineering Department, Korea Advanced Institute of Science and Technology (KAIST), Taejon, Republic of Korea. He received his MS from University of Colorado at Boulder in 1974 and his PhD from University of Texas at Austin in 1978. His research interests include robotics, precision machine system, and optical measurement. 